\title{
XMM-Newton observations of two black hole X-ray transients in quiescence
}

\author{
J.-M. Hameury ${ }^{1}$, D. Barret ${ }^{2}$, J.-P. Lasota ${ }^{3}$, J. E. McClintock ${ }^{4}$, K. Menou ${ }^{5, \star}$, C. Motch ${ }^{1}$, J.-F. Olive ${ }^{2}$, and N. Webb ${ }^{2}$ \\ 1 UMR 7550 du CNRS, Observatoire de Strasbourg, 11 rue de l'Université, 67000 Strasbourg, France \\ 2 Centre d'Etude Spatiale des Rayonnements, 9 Av. du Colonel Roche, BP 4346, 31028 Toulouse Cedex 4, France \\ ${ }^{3}$ UMR 7095 du CNRS, Institut d'Astrophysique de Paris, 98bis Boulevard Arago, 75014 Paris, France \\ ${ }^{4}$ Harvard-Smithsonian Center for Astrophysics, 60 Garden Street, Cambridge, MA 02138, USA \\ 5 Department of Astronomy, PO Box 3818, University of Virginia, Charlottesville, VA 22937, USA \\ Received 30 september 2002 / Accepted 22 November 2002
}

\begin{abstract}
We report on XMM-Newton observations of GRO J1655-40 and GRS 1009-45, which are two black hole X-ray transients currently in their quiescent phase. GRO J1655-40 was detected with a $0.5-10 \mathrm{keV}$ luminosity of $5.9 \times 10^{31} \mathrm{erg} \mathrm{s}^{-1}$ with $20 \%$ accuracy, taking into account both statistical errors and uncertainty on the distance. This luminosity is comparable to a previous Chandra measurement, but ten times lower than the 1996 ASCA value, most likely obtained when the source was not yet in a true quiescent state. Unfortunately, XMM-Newton failed to detect GRS 1009-45. A stringent upper limit of $8.9 \times 10^{30} \mathrm{erg} \mathrm{s}^{-1}$ was derived by combining data from the EPIC-MOS and PN cameras.

The X-ray spectrum of GRO J1655-40 is very hard as it can be fitted with a power law model of photon index $\sim 1.3 \pm 0.4$. Similarly hard spectra have been observed from other systems; these rule out coronal emission from the secondary or disk flares as the origin of the observed X-rays. On the other hand, our observations are consistent with the predictions of the disc instability model in the case that the accretion flow forms an advection dominated accretion flow (ADAF) at distances less than a fraction $(\sim 0.1-0.3)$ of the circularization radius. This distance corresponds to the greatest extent of the ADAF that is thought to be possible.
\end{abstract}

Key words. accretion, accretion discs - instabilities - black hole physics - X-ray: binaries stars: individual: GRO J1655-40 - stars: individual: GRS 1009-45

\section{Introduction}

Soft X-ray transients - SXTs (sometimes called X-ray novae) are semi-detached binaries in which the accreting (primary) star is a black hole $(\mathrm{BH})$ or a neutron star, and the mass-losing secondary is usually a late type star. These systems typically brighten in X-rays by as much as $10^{7}$ in a week and then decay back into quiescence over the course of a year. The maximum outburst luminosities $L_{\max }$ seen in SXTs are typically $\sim(0.2-1)$ of the Eddington luminosity $L_{\text {Edd }}$. Successive outbursts are usually separated by years to decades of quiescence (see e.g. Tanaka \& Shibazaki 1996; Chen et al. 1997, for reviews).

Quiescent states of SXTs are very interesting for at least two reasons. First, they provide the strongest evidence now available for the existence of stellar-mass black holes. The detection of the secondary in quiescence allows a determination of the mass function of the binary system, which is an absolute lower limit on the primary mass $M_{1}$. The mass function exceeds $3 M_{\odot}$ in eight systems; in five others, constraints on the inclination angle of the system and the secondary mass

Send offprint requests to: J.-M. Hameury,

e-mail: hameury@astro.u-strasbg.fr

^ Celerity Foundation Fellow. result in values for $M_{1}$ that are in the range 5-10 $M_{\odot}$ (Narayan et al. 2002). Thus there are now thirteen SXTs with primary masses that exceed the maximum stable mass of a neutron star $\left(\lesssim 3 M_{\odot}\right)$.

The second, more mundane reason why quiescent states are interesting is that quiescence provides a strong test of outburstcycle models. SXT outburst cycles are well described by the disc instability model (Dubus et al. 2001; see Lasota 2001 for a complete review of the disc instability model). In quiescence, the disc is non-steady (a property too often forgotten by too many authors). Its temperature and viscosity are low, and the disc is unable to transport all of the mass supplied by the secondary to the primary; the mass of the disc slowly builds up and the temperature rises finally to the hydrogen ionization temperature. At this moment, the disc becomes thermally and viscously unstable due to strong opacity variations. Propagating heat fronts bring the entire disc into a hot state; in this outburst state, the mass transfer rate is large, and the disc empties until it cannot sustain this regime any longer; it then returns to quiescence.

Even though the disc instability paradigm is widely accepted and its particular realizations are often successful (requiring sometimes additional assumptions, see e.g. Esin et al. 2000), the physics of the quiescent state is still rather 
Table 1. Main parameters of quiescent BH SXTs with known orbital periods $P_{\text {orb. }} M_{1}$ is the primary mass (from Orosz 2002 and references therein), and $n_{21}$ the hydrogen column density in units of $10^{21} \mathrm{~cm}^{-2}$, generally inferred from optical extinction. The luminosity refers to the 0.5-10 keV energy range; $\alpha$ is the power law photon index. The orbital periods and masses have been taken from Narayan et al. (2002) and references therein, and updated from Orosz (2002); data for GRO J1655-40 are from Beer \& Podsiadlowski (2002). The dividing line separates somewhat arbitrarily systems with short and long orbital periods.

\begin{tabular}{|c|c|c|c|c|c|c|c|c|}
\hline & $\begin{array}{l}P_{\text {orb }} \\
(\mathrm{hr})\end{array}$ & $\begin{array}{c}D \\
(\mathrm{kpc})\end{array}$ & Ref. & $\begin{array}{c}M_{1} \\
\left(M_{\odot}\right)\end{array}$ & $n_{21}$ & $\log L_{X}$ & $\alpha$ & Reference (X-rays) \\
\hline XTE J1118+480 & 4.1 & $1.8 \pm 0.6$ & 1 & $6.8 \pm 0.4$ & 0.1 & & & \\
\hline GRO J0422+32 & 5.1 & $2.6 \pm 0.5$ & 2 & $4.3 \pm 0.7$ & 1.6 & 30.9 & - & Chandra (13) \\
\hline GRS 1009-45 & 6.9 & $5 \pm 1.5$ & 3 & $4.2 \pm 0.6$ & 1.1 & $<30.9$ & & XMM-Newton - this paper \\
\hline A0620-00 & 7.8 & $1.1 \pm 0.1$ & 4 & $10.8 \pm 2.1$ & $\begin{array}{c}1.9 \\
0.16\end{array}$ & $\begin{array}{l}30.6 \\
30.7\end{array}$ & $2.07_{-0.19}^{+0.28}$ & $\begin{array}{l}\text { Chandra (14) } \\
\text { ROSAT (15) }\end{array}$ \\
\hline GS $2000+25$ & 8.3 & $2.7 \pm 0.8$ & 5 & $7.5 \pm 0.3$ & 11 & 30.4 & - & Chandra (13) \\
\hline XTE J1859+226 & 9.2 & $6.3 \pm 1.7$ & 6 & $9.8 \pm 2.2$ & & & & \\
\hline Nova Mus 1991 & 10.4 & $5.5 \pm 1.0$ & 7 & $7.3 \pm 0.9$ & 2.6 & 31.6 & $1.6 \pm 0.4$ & XMM-Newton (16) \\
\hline Nova Oph 1977 & 12.5 & $8.0 \pm 2.4$ & 5 & $7.0 \pm 1.3$ & 2.8 & $<33.0$ & & ROSAT (17) \\
\hline $4 U 1543-47$ & 27.0 & $9.1 \pm 1.1$ & 8 & $9.4 \pm 1.0$ & 3.5 & $<31.5$ & & Chandra (13) \\
\hline XTE J1550-564 & 37.0 & $5.3 \pm 2.3$ & 9 & $9.6 \pm 1.2$ & 3.9 & 33.0 & $1.35 \pm 0.25$ & Chandra (14) \\
\hline \multirow[t]{3}{*}{ GRO J1655-40 } & 62.9 & $3.2 \pm 0.2$ & 10 & $6.3 \pm 0.3$ & 6.7 & 31.5 & $1.47 \pm 0.4$ & Chandra (14) \\
\hline & & & & & & 31.8 & $1.30 \pm 0.40$ & XMM-Newton - this paper \\
\hline & & & & & $<3$ & 33.1 & $0.7_{-0.4}^{+0.21}$ & $\operatorname{ASCA}(18)$ \\
\hline V4641 Sgr & 67.6 & $10.0 \pm 2.5$ & 11 & $7.1 \pm 0.3$ & 0.5 & & & \\
\hline \multirow[t]{3}{*}{ V404 Cyg } & 155.3 & $3.5 \pm 1$ & 12 & $11.7 \pm 1.7$ & 5.4 & 33.6 & $1.55 \pm 0.07$ & Chandra (14) \\
\hline & & & & & $11_{-4}^{+3}$ & 33.1 & $2.1_{-0.3}^{+0.3}$ & ASCA (19) \\
\hline & & & & & 10 & 33.0 & $1.9_{-0.3}^{+0.6}$ & Beppo-SAX (20) \\
\hline
\end{tabular}

References: (1) McClintock et al. (2001); (2) Esin et al. (1998); (3) Barret et al. (2000); (4) Gelino et al. (2001a); (5) Barret et al. (1996); (6) Hynes et al. (2002); (7) Orosz et al. (1996), Gelino et al. (2001b); (8) Orosz et al. (1998); (9) Orosz et al. 2002); (10) Hjellming \& Rupen (1995); (11) Orosz et al. (2001) (12) Shahbaz et al. (1994); (13) Garcia et al. (2001); (14) Kong et al. (2002); (15) Narayan et al. (1996); (16) Sutaria et al. (2002); (17) Verbunt et al. (1994); (18) Asai et al. (1998); (19) Narayan et al. (1997a); (20) Campana et al. (2001).

controversial. As a prime example, the origin of "viscosity" in this state is not really known (see e.g. Gammie \& Menou 1998; Menou 2002; Lasota 2002), and according to the simplest version of the disc-instability model (the version originally used to explain dwarf-nova outbursts; Lasota 2001) the very long SXT recurrence times require unusually low values of the viscosity parameter $\alpha$. However, Dubus et al. (2001) showed that the combined effects of disc irradiation during outbursts (King $\&$ Ritter 1998) and disc truncation during quiescence (Menou et al. 2000) result quite effortlessly in long recurrence times for standard values of the viscosity parameter, making the disc instability model for SXTs a working and testable hypothesis.

Narayan et al. (1996; see also Lasota et al. 1996 and Narayan et al. 1997a) noticed that spectra of quiescent SXTs cannot be produced by geometrically thin, optically thick accretion discs and suggested that the inner flow in such systems forms an advection dominated accretion flow ADAF. In any case, according to the disc instability model a disc extending down to the neutron star surface or the last stable orbit around a black hole, can be in a cold, neutral state everywhere only for vanishingly low accretion rates close to the central object (Lasota 1996): the maximum accretion rate onto the compact object would be $\sim 4000\left(M_{1} / M_{\odot}\right)^{1.77}\left(r_{\text {in }} / r_{\mathrm{s}}\right)^{2.65} \mathrm{~g} \mathrm{~s}^{-1}$, where $r_{\mathrm{s}}$ is the Schwarzschild radius (Hameury et al. 1998), much too low to produce the X-ray luminosity observed in quiescent SXTs. On the other hand the ADAF model reproduces well the observed luminosities, spectra (Narayan et al. 1997a;
Quataert \& Narayan 1999) and observed delays between the rises to outburst in optical and X-rays (Hameury et al. 1997).

As first pointed out by Narayan et al. (1997b), the presence of the radiatively inefficient ADAFs in quiescent SXTs allows one to compare black holes with compact bodies endowed with material surfaces. Because bodies such as neutron stars must re-emit the heat left over in the accretion flow accumulating at their surface they should be brighter than black holes accreting at the same rate since in this case the residual energy is lost forever past the event horizon. X-ray observations of quiescent SXTs showed that this is indeed the case (Narayan et al. 1997b; Menou et al. 1999b; Garcia et al. 2001). However, the ADAF origin of X-rays in quiescent SXTs has been questioned by several authors (see e.g. Bildsten \& Rutledge 2001; Nayakshin \& Svensson 2001). High quality X-ray observations can solve this controversy.

In this paper, we report on XMM-Newton observations of GRO J1655-50 (AO-1 GTO) and GRS 1009-45 (AO-1 GO).We briefly describe previous observations of these transients in Sect. 2; we present our observations in Sect. 3, and we discuss their implications in Sect. 4.

\section{Earlier observations}

X-ray observations of quiescent SXTs prior to XMM-Newton and Chandra have yielded only marginal detections or upper limits, with very poor constraints on the spectral shape. Among 
the 13 BHSXTs with known orbital periods, A 0620-00 was the only one to be detected by ROSAT (McClintock et al., 1995); ASCA detected GRO J1655-40 (Asai et al. 1998; Ueda et al. 1998) and V404 Cyg (Narayan et al. 1997a). Finally, among the 5 BHSXTs observed by BeppoSAX (Campana et al. 2001), only V404 Cyg has been detected with a 1-10 keV unabsorbed luminosity of $\sim 10^{33} \mathrm{erg} \mathrm{s}^{-1}$.

XMM-Newton and Chandra have already qualitatively changed this situation, as can be clearly seen from Table 1, which gives spectral data for most of the 13 SXTs with known orbital period, and with primary masses exceeding $3 M_{\odot}$. The $\mathrm{X}$-ray luminosities have been computed with the most recent and accurate distance estimates and associated uncertainties. When no estimate on the error on distance was available, we arbitrarily assumed it to be $30 \%$ for the distances given by Barret et al. (1996; compare relative measurements in their Table 1), or $50 \%$ in all other cases.

It is now evident that many quiescent SXTs have been detected with luminosities in the range $10^{31}-10^{33} \mathrm{erg} \mathrm{s}^{-1}$, the faintest being those with the shortest orbital periods. It is also clear that SXTs have very hard spectra with values of the power law spectral index that are often less than 2. Or equivalently, their spectra can be fitted by a bremsstrahlung model with temperatures larger than $10 \mathrm{keV}$ (Kong et al. 2002).

\section{XMM-Newton observations and analysis}

\subsection{GRO J1655-40}

GRO J1655-40 was observed by XMM-Newton on August 30, 2001 with a $40 \mathrm{ksec}$ exposure time. Due to a strong solar flare, only 19 ks could be used; yet, GRO J1655-40 was clearly detected as one of the brightest field sources, at a position coincident with that of the optical counterpart (within $0.3^{\prime \prime}$ ), with a total source count rate as determined by the automatic pipeline analysis of $1.09 \times 10^{-2} \pm 1.8 \times 10^{-3}$.

SAS version 5.3 was used to calculate the source and background spectra obtained by extracting data from a circle of approximately $20^{\prime \prime}$ radius centered on the nominal source position, and from an annulus with inner and outer radii $\sim 20^{\prime \prime}$ and $\sim 100^{\prime \prime}$ respectively. 74,73 and 260 counts were detected in the $20^{\prime \prime}$ radius circle in the MOS1, MOS2 and PN cameras respectively; 618 (MOS1), 403 (MOS2) and 1225 (PN) counts were detected in the larger area used to determine the background rate. The spectra were analyzed with Xspec v11. Due to the small number of counts, in particular in the MOS cameras, the C-statistics (Cash 1979) was used to determine the best fits and errors; channels were grouped in such a way that there were at least 5 counts in each bin. The three cameras, MOS1, MOS2, and PN were used simultaneously to determine the best fit parameters.

Table 2 summarizes our results. A power law is a good fit to the results, yielding column density $n_{\mathrm{H}}=1.0_{-0.7}^{+1.2} 10^{22} \mathrm{~cm}^{-2}$, a power law index $\alpha=1.54_{-0.72}^{+1.02}$, and normalization $K=7.1 \times 10^{-6}$, poorly constrained because of the large error in $\alpha$. If one uses a hydrogen column density fixed to the value of $n_{\mathrm{H}}=6.7 \times 10^{21} \mathrm{~cm}^{-2}$ determined by optical observations (Hynes et al. 1998), one obtains $\alpha=1.30_{-0.42}^{+0.34}$, and
GRO J1655-40

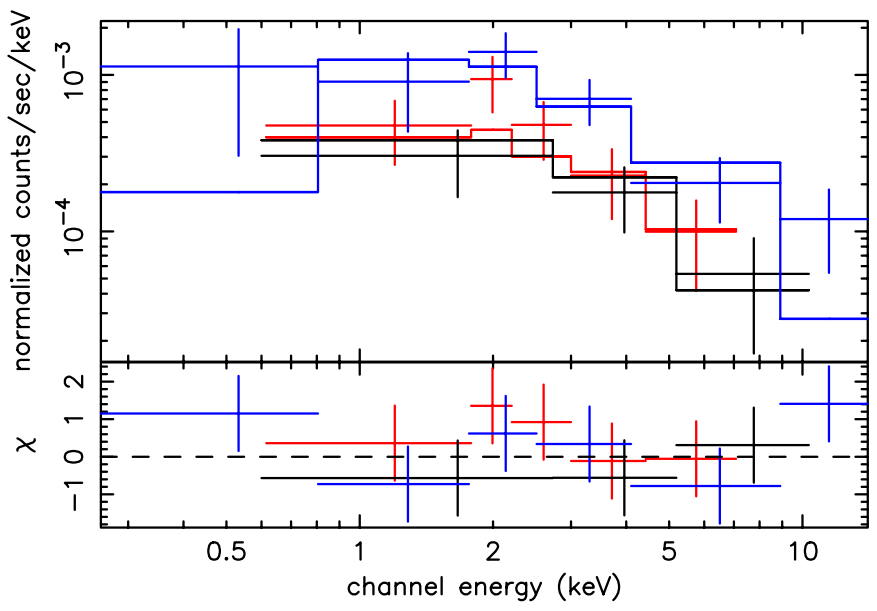

Fig. 1. Top: XMM-Newton spectrum of GRO J1655-40, fitted by a power law. Bottom: residuals after model subtraction from the data, in units of $1 \sigma$. The pn data are in blue, the MOS data in black and red. (This figure is available in color in electronic form.)

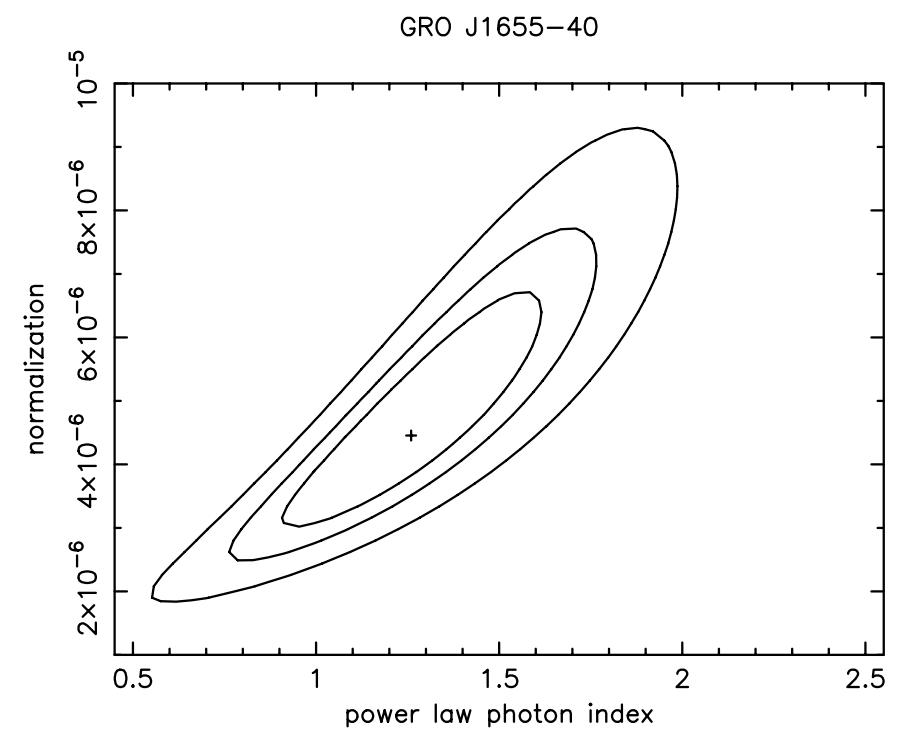

Fig. 2. Confidence level at 68,90 and $99 \%$ of the fit given in Fig. 1. $n_{\mathrm{H}}$ is kept fixed at $6.7 \times 10^{21} \mathrm{~cm}^{-2}$.

$K=4.8_{-1.9}^{+2.0} \times 10^{-6}$. All errors given here are $90 \%$ confidence limits. Figure 1 shows the EPIC spectrum for all three instruments, as well as the best fit and residuals, in units of 1 sigma. Note that these residuals, although giving a rough estimate of the quality of the fit, cannot be used to determine the goodnessof-fit by computing a $\chi^{2}$, since the C-stat has been used. Note also that, for the sake of clarity, data in Fig. 1 have been rebinned, so that more data points were used for performing the spectral fitting. Figure 2 gives the 68.3, 90 and $99 \%$ confidence contours obtained for the power law index and normalization.

The total absorbed flux is then $3.96_{-0.83}^{+0.66} \times 10^{-14} \mathrm{erg} \mathrm{cm}^{-2} \mathrm{~s}^{-1}$ in the $0.5-10 \mathrm{keV}$ range (90\% confidence error bars), corresponding to an unabsorbed flux of $4.81_{-1.00}^{+0.80} \times 10^{-14} \mathrm{erg} \mathrm{cm}^{-2} \mathrm{~s}^{-1}$ and unabsorbed luminosity of $5.9 \times 10^{31} \mathrm{erg} \mathrm{s}^{-1}$ for a distance of $3.2 \mathrm{kpc}$. 
Table 2. Best fitting spectral parameters for GRO J1655-40. $F_{0.5-10}$ is the $0.5-10 \mathrm{keV}$ unabsorbed flux. The goodness is the probability that simulated data give a better $\mathrm{C}$ statistic than real data.

\begin{tabular}{lccccccc}
\hline \hline Model & $\begin{array}{c}n_{\mathrm{H}} \\
\left(10^{21} \mathrm{~cm}^{-2}\right)\end{array}$ & $\alpha$ & $\begin{array}{c}k T \\
(\mathrm{keV})\end{array}$ & C statistic & d.o.f. & goodness & $\begin{array}{c}F_{0.5-10} \\
\left(0^{-14}\left(\mathrm{erg} \mathrm{cm}^{-2} \mathrm{~s}^{-1}\right)\right.\end{array}$ \\
\hline power law & $10.0_{-0.7}^{+1.2}$ & $1.54_{-0.72}^{+1.02}$ & & 63.8 & 72 & 0.12 & 5.3 \\
power law & 6.7 (fixed) & $1.30_{-0.41}^{+0.34}$ & & 64.5 & 73 & 0.11 & 4.8 \\
bremsstrahlung & 6.7 (fixed) & & $>9$ & 64.5 & 73 & 0.12 & 4.1 \\
\hline
\end{tabular}

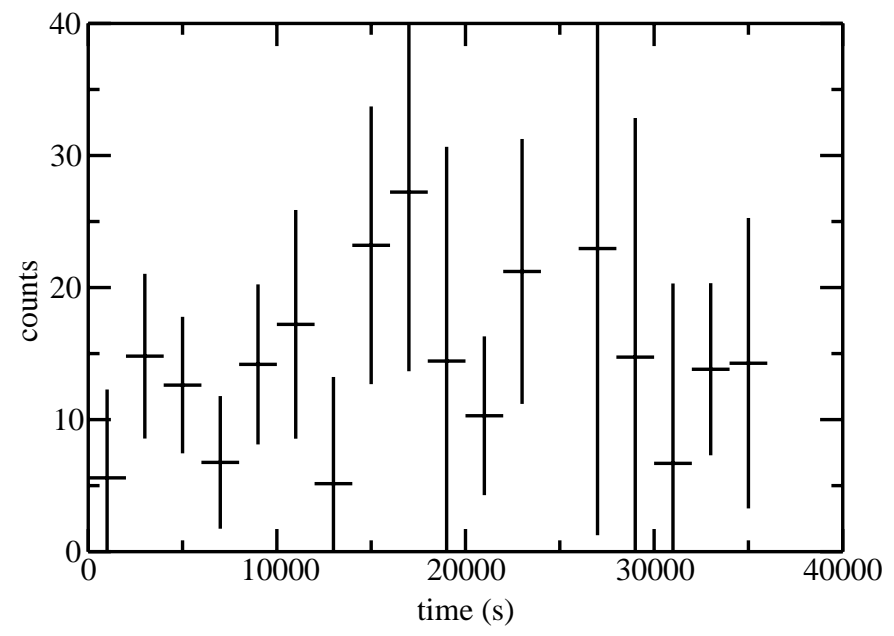

Fig. 3. Net count rate per $2000 \mathrm{~s}$ bins for GRO J1655-40 in the PN camera. Large errors are produced by the strong solar flare.

We also fitted the spectrum with a thermal bremsstrahlung. As expected for a hard spectrum with such a photon index, the fit is good, with a formal best fit value of the temperature $k T=$ $110 \mathrm{keV}$, indeed unphysical, and very poorly constrained; the $90 \%$ confidence level lower limit on $k T$ is $9 \mathrm{keV}$, and the $99 \%$ confidence level lower limit is $4.1 \mathrm{keV}$.

A search for variability yielded negative results for significant variations on time scales of hours. Figure 3 shows the source intensity as a function of time, with $2000 \mathrm{~s}$ time bins; the data are consistent with a constant source. The data quality is quite poor during the solar flare that occurs after the first $10 \mathrm{ks}$, and lasts about $25 \mathrm{ks}$, with shorts lulls.

\subsection{GRS $1009-45$}

GRS 1009-45 was observed on May 30, 2002, during a $20 \mathrm{ksec}$ exposure. The source was not detected by the automatic pipeline processing system. Assuming a power law spectrum with photon index 2 , and a hydrogen column density $n_{\mathrm{H}}=$ $1.1 \times 10^{21} \mathrm{~cm}^{-2}$, as given by optical observations (della Valle et al. 1997), and analyzing simultaneously all three cameras together, we can set an upper limit for the $0.5-10 \mathrm{keV}$ unabsorbed flux of $3 \times 10^{-15} \mathrm{erg} \mathrm{cm}^{-2} \mathrm{~s}^{-1}$ (95\% confidence), corresponding to an upper limit on the luminosity of $8.9 \times 10^{30} \mathrm{erg} \mathrm{s}^{-1}$ for a distance of $5 \mathrm{kpc}$. To determine this upper limit, we fixed the photon index and column density in Xspec and calculated the goodness-of-fit for various normalization factors, with the $\chi^{2}$ statistics (using the C-statistics yields an almost identical upper limit, as expected). The data are compatible with a zero flux (we obtain a goodness-of-fit of $63 \%$ ); we can set an upper limit on the flux of $3 \times 10^{-15} \mathrm{erg} \mathrm{cm}^{-2} \mathrm{~s}^{-1}$, for which the goodness-of-fit is 0.95 , i.e. in only $5 \%$ of the simulated cases did we find a $\mathrm{C}$ statistic value worse than the value actually obtained. This upper limit is almost independent of the assumed photon power law index.

\section{Discussion}

It has been suggested (Bildsten \& Rutledge 2001) that X-rays detected from quiescent black-hole SXTs are not produced by accretion, but are instead produced in the secondaries' hot stellar coronae. There is now growing evidence that this is not the case because the X-ray flux is too large (especially the $L_{\mathrm{X}}$ relative to the bolometric luminosity of the secondary) and/or because the spectrum is too hard to be emitted by a stellar corona (Kong et al. 2002). As we shall see, the flaring disc model also faces similar difficulties, whereas the ADAF explanations can still account for the observations.

\subsection{Spectra}

The spectra of all black hole transients appear to be quite hard, GRO J1655-40 being the hardest, and A0620-00 having the steepest power law index; fits using bremsstrahlung models also lead to very high temperatures. This confirms previous findings (Kong et al. 2002) that coronal emission from the secondary is ruled out. Einstein surveys of late type stars have shown that the coronal temperatures of isolated stars do not significantly exceed $1 \mathrm{keV}$, and reach at most a few $\mathrm{keV}$ in the case of giant RS CVn stars (Schmitt 1990). (Coronal emission is additionally ruled out by the high $\mathrm{X}$-ray luminosities; see below.)

It is also most unlikely that such spectra could be produced by emission from magnetic loops above the disc (see e.g. Nayakshin \& Svensson 2001) that would very probably result in spectra similar to those of stellar coronae. Moreover, this model implies that the accretion disc of quiescent dwarf novae should emit a comparable soft X-ray flux; these systems are indeed X-ray bright, with luminosities in the range $10^{30}-10^{32} \mathrm{erg} \mathrm{s}^{-1}$, but they have deep eclipses indicating that the X-ray emitting region is very close to the white dwarf; the residual X-ray luminosity during eclipses is very low (e.g. of the order of $9 \times 10^{28} \mathrm{erg} \mathrm{s}^{-1}$ for OY Car, Ramsay et al. 2001). 


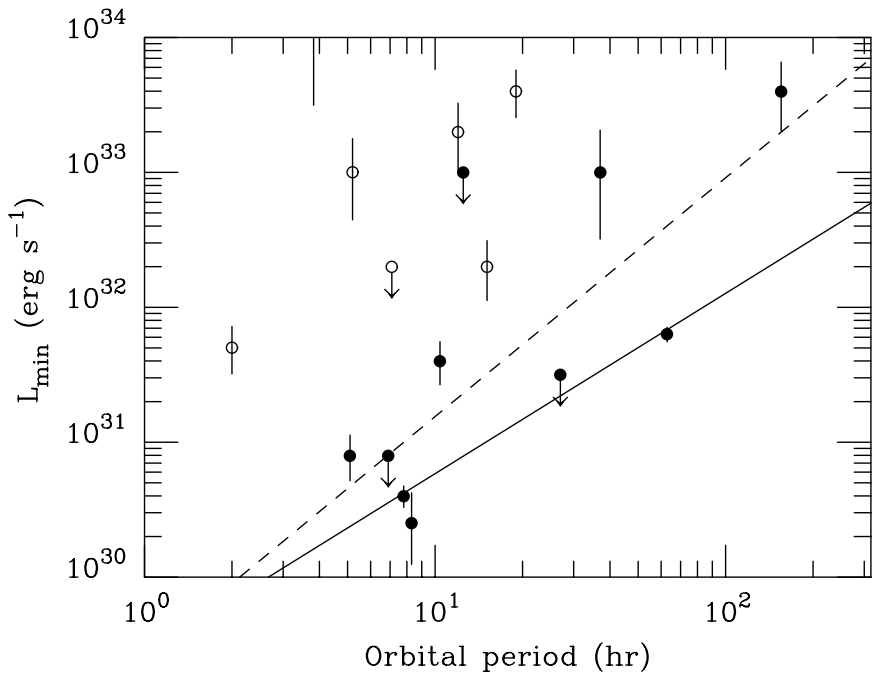

Fig. 4. Quiescent luminosities of SXTs containing black holes (filled circles: Chandra and XMM-Newton data from Table 1), or a neutron star (open circles: data from Narayan et al. 2002, except for SAX J1808.4-3658 for which we used XMM-Newton data of Campana et al. 2002, who found the source in deeper quiescence than Wijnands 2002a). Also shown are the maximum coronal X-ray luminositiy (the "FGM-limit", Flemming et al. 1989) for a $1 M_{\odot}$ secondary (solid line), and (dashed line) the flux predicted by Lasota (2000).

\subsection{Luminosities}

Figure 4 shows the luminosities of SXTs containing black holes compared to those in which the accreting object is a neutron star. We plot the quiescent luminosity (in erg $\mathrm{s}^{-1}$ ) as a function of the orbital period of the binary. Here, the data for $\mathrm{BH}$ systems have been taken from Table 1; when several measurements were available, we selected Chandra or XMM-Newton values (for GRO J1655-40, the XMM-Newton value given in this paper is used). The NS data are from Narayan et al. (2002). The error bars reflect uncertainties on distance only, which are rather large, and are usually the most important contributor to the error on luminosity. For SAX J1808.4-3658, we assumed $d=2.5 \pm 0.5 \mathrm{kpc}$ (in't Zand et al. 2001); for EXO 0748$676, d=10 \mathrm{kpc}$ with an arbitrary error of $5 \mathrm{kpc}$ (Gottwald et al. 1986); for $4 \mathrm{U} 2129+47, d=6 \pm 2 \mathrm{kpc}$ (Nowak et al. 2002); for MXB 1659-298, $d=12 \pm 2.5 \mathrm{kpc}$ (Wijnands 2002b); for $\mathrm{H} 1608-52, d=3.6 \mathrm{kpc}$ with a typical error of order $1 \mathrm{kpc}$ (Wachter et al. 2002); for Cen X-4, $d=1.2 \pm 0.3 \mathrm{kpc}$ (Chevalier et al. 1989; for Aql X-1, $d=5 \pm 1 \mathrm{kpc}$ (Rutledge et al. 2001). Our result confirms the well established fact that systems containing black holes are, for a given orbital period, much dimmer than those containing a neutron star (i.e. in which X-ray bursts have been detected), a property predicted by models in which the inner disc is truncated and replaced by an ADAF close to the black hole (Menou et al. 1999b).

The $L_{\mathrm{X}}-P_{\text {orb }}$ plot was proposed by Lasota \& Hameury (1998) to assure, when one compares black-hole systems with those containing neutron stars, that both types of systems have comparable accretion rates at the outer limit of the ADAF. Sutaria et al. (2002) pointed out recently that for various reasons one should expect a "greater spread" of $\dot{M}$ (the masstransfer rate) at a given $P_{\text {orb }}$. This is obviously true. However, if SXT outbursts are due to a dwarf-nova-type disc instability, then in quiescence the accretion rate at the truncation radius depends only weakly on the mass-transfer rate (e.g. Menou et al. 1999a; Lasota 2000; Dubus et al. 2001; but see also Menou 2002 who discusses uncertainties linked to our poor understanding of viscosity in the quiescent state and a possible alternative solution). Indeed, the accretion rate at the transition radius can be written as (Lasota 2000)

$\dot{M}_{\mathrm{tr}} \approx 2.4 \times 10^{15}\left(\frac{M}{7 M_{\odot}}\right)^{1.77}\left(\frac{R_{\mathrm{tr}}}{10^{4} R_{\mathrm{G}}}\right)^{2.65} \mathrm{~g} \mathrm{~s}^{-1}$.

In addition, as discussed in Lasota (2000) the prescription for the transition radius proposed by Menou et al. (1999a), i.e.

$R_{\mathrm{tr}}=f_{\mathrm{t}} R_{\text {circ }}, \quad f_{\mathrm{t}}<0.48$,

where the circularization radius $R_{\text {circ }}$ can be approximated by (Frank et al. 2002)

$\frac{R_{\text {circ }}}{a}=(1+q)[0.5-0.227 \times \log q]^{4}$,

transforms Eq. (1) into

$\dot{M}_{\mathrm{ADAF}} \approx 1.6 \times 10^{18} f_{\mathrm{t}}^{2.65} P_{\text {day }}^{1.77} \mathrm{~g} \mathrm{~s}^{-1}$

where $\dot{M}_{\mathrm{ADAF}}$ is the rate at which matter enters the ADAF from the cold quiescent, non-steady disc. This rate is independent of the primary's mass.

To obtain Eq. (1) one assumes that according to the disc instability model the quiescent disc is non-steady and $R_{\mathrm{tr}} \ll$ $R_{\text {out }}$, where $R_{\text {out }}$ is the outer radius of the geometrically thin accretion disc. The pre-Chandra and pre-XMM-Newton data (for three systems) satisfied a $L_{\mathrm{X}} \sim P_{\mathrm{orb}}^{1.77}$ relation (dashed line in Fig. 4), the slope of which suggested that it might result from Eq. (4) (Lasota 2000).

The new Chandra and XMM-Newton observations show a more complicated picture. First, the quiescent X-ray luminosity of GRO J1655-40 is now an order of magnitude lower than it was when observed by ASCA. In fact the quiescent luminosities of all three systems (A0620-00, GRO J1655-40 and V404 Cyg) used by Lasota (2000) to determine the $\sim 1.77$ slope of the $L_{\mathrm{X}}-P_{\text {orb }}$ relation have been revised significantly (see Table 1 and discussion below). In the case of A0620-00 and V404 Cyg there is little doubt that they are in true quiescence. Among the new sources, XTE J1550-564 was observed between two outbursts (see Kong et al. 2002 and references therein) so that this system was very unlikely to be in its true quiescent state during the Chandra observations.

The quiescent luminosity might still be described by Eq. (4) but with $f_{t}$ depending on the system. Such a conclusion is not unexpected since Menou et al. (1999a) have already noticed that although $f_{\mathrm{t}} \approx 0.25$ for most systems, V404 Cyg required $f_{\mathrm{t}} \lesssim 0.1$. The value of $f_{\mathrm{t}}$ should be predicted by the model describing the "evaporation" of an accretion disc into an ADAF, but we lack such a model.

\subsection{Variability}

The flux we obtained for GRO J1655-40 is consistent with that found by Chandra (Kong et al. 2002), the difference being of 
order of $50 \%$ for the unabsorbed flux in the $0.2-10 \mathrm{keV}$ range, and can be essentially accounted for by statistical fluctuations; we do not find significant short time scale variability, which means that these sources are relatively stable. However, both XMM-Newton and Chandra flux measurements are one order of magnitude below that of ASCA (Asai et al. 1998; Ueda et al. 1998). This observation, however, was performed a month before the 1996 outburst and roughly 9 months after the previous (August 1995) eruption. Clearly, the system was not in its quiescent state as it takes more than 30 years (the recurrence time) to refill the disc emptied by the outburst. Matter was then transferred from the secondary at a rate much higher than during the true quiescence (Esin et al. 2000).

Short time scale variability has been detected transients during quiescence containing black holes, such as V404 Cyg (Wagner et al. 1994; Kong et al. 2002), as well as those containing neutron stars, such as Aql X-1 (Rutledge et al. 2002). Such variability is not a surprise, but cannot be significantly constrained in our data due to the low count rate.

\section{Conclusions}

We have observed and detected GRO J1655-40 during a $40 \mathrm{ksec}$ XMM-Newton observation, with a luminosity of $5.9 \times$ $10^{31} \mathrm{erg} \mathrm{s}^{-1}$; GRS 1009-45 was undetected, leading to a relatively small upper limit on luminosity of $8.9 \times 10^{30} \mathrm{erg} \mathrm{s}^{-1}$ for a distance of $5 \mathrm{kpc}$. These observations are consistent with the disc instability model if the accretion disc is truncated at a radius of $0.1-0.3$ times the circularization radius, where the accretion flow forms an ADAF. The spectrum appears to be quite hard, much harder than one would expect from coronal emission from the secondary star or from the accretion disc itself. The quality of the data is however not sufficient to constrain and distinguish between various types of optically thin flows in the vicinity of the black hole.

There is no sign of variability on a time scale of hours, and the flux measured by XMM-Newton from GRO J1655-40 is consistent with the Chandra value (Kong et al. 2002), and about 10 times lower than that which was measured with ASCA by Asai et al. (1998) and Ueda et al. (1998) between two outbursts, indicating that the system was not fully in quiescence at that time, and that the mass transfer rate from the secondary was high.

Acknowledgements. We are grateful to R. Narayan for discussions and comments on the manuscript. This work was supported in part by a grant from the Centre National d'Etudes Spatiales.

\section{References}

Asai, K, Dotani, T., Hoshi, R., et al. 1998, PASJ, 50, 611 Barret, D., McClintock, J. E., \& Grindlay, J. E. 1996, ApJ, 473, 963

Barret, D., Olive, J. F., Boirin, L., et al. 2000, ApJ, 533, 329

Beer, M. E., \& Podsiadlowski, P. 2002, MNRAS, 331, 351

Bildsten, L., \& Rutledge, R. E. 2001, ApJ, 541, 908

Campana, S., Parmar, A. N., \& Stella, L. 2001, A\&A, 372, 241

Campana, S., Stella, L., Gastaldella, et al. 2002, ApJ, 575, L15

Cash, W. 1979, ApJ, 228, 939

Chen, W., Schrader, C. R., \& Livio, M. 1997, ApJ, 491, 312
Chevalier, C., Ilovaisky, S. A., van Paradijs, J., Pedersen, H., \& van der Klis, M. 1989, A\&A, 210, 114

della Valle, M., Benetti, S., Cappellaro, E., \& Wheeler, C. 1997, A\&A, 318,179

Dubus, G., Hameury, J. M., \& Lasota, J. P. 2001, A\&A, 373, 251

Esin, A. A., Narayan, R., Cui, W., Grove, J. E., \& Zhang, S.-N. 1998, ApJ, 505, 854

Esin, A. A., Lasota, J.-P., \& Hynes, R. I. 2000, A\&A, 354, 987

Fleming, T. A., Gioia, I. M., \& Maccacaro, T. 1989, ApJ, 340, 1011

Frank, J., King, A., \& Raine, D. 2002, Accretion power in astrophysics, 3rd ed. (Cambridge University Press)

Gammie, C. F., \& Menou, K. 1998, ApJ, 492, L75

Garcia, M. R., McClintock, J. E., Narayan, R., et al. 2001, ApJ, 553, L47

Gelino, D. M., Harrison, T. E., \& Orosz, J. A. 2001, AJ, 122, 2668

Gelino, D. M., Harrison, T. E., \& McNamara, B. J. 2001, AJ, 122, 971

Gottwald, M., Haberl, F., Parmar, A. N., \& White, N. E. 1986, ApJ, 308,213

Hameury, J.-M., Lasota, J.-P., McClintock, J. E., \& Narayan, R. 1997, ApJ, 489, 234

Hameury, J.-M., Menou, K., Dubus, G., Lasota, J.-P., \& Huré, J.-M. 1998, MNRAS, 298, 1048

Hjellming, R. M., \& Rupen, M. P. 1995, Nature, 375, 464

Hynes, R. I., Haswell, C. A., Shrader, C. R., et al. 1998, MNRAS, 300, 64

Hynes, R. I., Haswell, C. A., Chaty, S., Shrader, C. R., \& Cui, W. 2002, MNRAS, 331, 169

in't Zand, J. J. M., Cornelisse, R., Kuulkers, E., et al. 2001, A\&A, 372, 916

King, A. R., \& Ritter, H. 1998, MNRAS, 293, L42

Kong, A. K. H., McClintock, J. E., Garcia, M. R., Murray, S. S., \& Barret, D. 2002, ApJ, 570, 277

Lasota, J.-P. 1996, IAU Symp., 165, Compact Stars in Binaries, 165, 43

Lasota, J.-P. 2000, A\&A, 360, 575

Lasota, J.-P. 2001, New AR, 45, 449

Lasota, J.-P. 2002, in The Physics of Cataclysmic Variables and Related Objects, ed. B. T. Gänsicke, K. Beuermann, \& K. Reinsch, ASP Conf. Ser., 261, 397

Lasota, J.-P., \& Hameury, J.-M. 1998, in Accretion Processes in Astrophysical Systems: Some Like it Hot!, ed. S. Holt, \& T. Kallman (New York: AIP), AIP Conf. Proc., 431, 351

Lasota, J.-P., Narayan, R., \& Yi, I. 1996, A\&A, 314, 813

McClintock, J. E., Horne, K., \& Remillard, R. A. 1995, ApJ, 442, 358

McClintock, J. E., Garcia, M. R., Caldwell, N., et al. 2001, ApJ, 551, L147

Menou, K. 2002, in The physics of cataclysmic variables and related objects, ed. B.T. Gänzicke, K. Beuermann, \& K. Reinsch, ASP Conf. Ser., 261, 387

Menou, K., Narayan, R., \& Lasota, J. P. 1999, ApJ, 513, 811

Menou, K., Esin, A. A., Narayan, R., et al. 1999, ApJ, 520, 276

Menou, K., Hameury, J. M., Lasota, J. P., \& Narayan, R. 2000, MNRAS, 314, 498

Narayan, R., McClintock, J. E., \& Yi, I. 1996, ApJ, 457, 821

Narayan, R., Barret, D., \& McClintock, J. E. 1997, ApJ, 482, 448

Narayan, R., Garcia, M. R., \& McClintock, J. E. 1997, ApJ, 478, L79

Narayan R., Garcia, M. R., \& McClintock, J. E. 2002, in Proc. IX Marcel Grossmann Meeting, ed. V. Gurzadyan, R. Jantzen, \& R. Ruffini (Singapore: World Scientific), in press [astro-ph/0107387]

Nayakshin, S., \& Svensson, R. 2001, ApJ, 551, L67

Nowak, M. A., Heinz, S., \& Begelman, M. C. 2002, ApJ, 573, 778 
Orosz, J. A. 2002, in A massive star odyssey from main sequence to supernova, Proc. IAU Symp., 212, ed. K. A. van der Hucht, A. Herrero, \& C. Esteban (San Francisco: ASP), in press

Orosz, J. A., Bailyn, C. D., McClintock, J. E., \& Remillard, R. A. 1996, ApJ, 468, 380

Orosz, J. A., Jain, R. K., Bailyn, C. D., McClintock, J. E., \& Remillard, R. A. 1998, ApJ, 499, 375

Orosz, J. A., Kuulkers, E., van der Klis, M., et al. 2001, ApJ, 555, 489

Orosz, J. A., Groot, P. J., van der Klis, M., et al. 2002, ApJ, 568, 845

Quataert, E., \& Narayan, R. 1999, ApJ, 520, 298

Ramsay, G., Poole, T., Mason, K., et al. 2001, A\&A, 365, L288

Rutledge, R. E., Bildsten, L., Brown, E. F., Pavlov, G. G., \& Zavlin, V. E. 2001, ApJ, 559, 1054

Rutledge, R. E., Bildsten, L., Brown, E. F., Pavlov, G. G., \& Zavlin, V. E. 2002, ApJ, 577, 346

Schmitt, J. H. M. M., Collura, A., Sciortino, S., et al. 1990, ApJ, 365, 704
Shahbaz, T., Ringwald, F. A., Bunn, J. C., et al. 1994, MNRAS, 271, L10

Sutaria, F. K., Kolb, U., Charles, P., et al. 2002, A\&A, 391, 993

Tanaka, Y., \& Shibazaki, N. 1996, ARA\&A, 34, 607

Ueda, Y., Inoue, H., Tanaka, Y., et al. 1998, ApJ, 492, 782

Verbunt, F., Belloni, T., Johnston, H. M., van der Klis, M., \& Lewin, W. H. G. 1994, A\&A, 285, 903

Wagner, R. M., Starrfield, S. G., Hjellming, R. M., Howell, S. B., \& Kreidl, T. J. 1994, ApJ, 429, L25

Wachter, S., Hoard, D. W., Bailyn, C. D., Corbel, S., \& Kaaret, P. 2002, ApJ, 568, 901

Wijnands, R. 2002a, ApJL, submitted [astro-ph/0207102]

Wijnands, R. 2002b, in X-rays at sharp focus: Chandra science Symp., ed. E. Schegel, \& S. Vrtilek, ASP Conf. Ser., 262, 235 